\title{
Cesarean Scar Pregnancy Registry: an international research platform
}

Andrea Kaelin Agten, $\mathrm{MD}^{1}$, Ana Monteagudo, $\mathrm{MD}^{2}$, Ilan E. Timor-Tritsch, $\mathrm{MD}^{3}$, Basky Thilaganathan, MRCOG, MD, $\mathrm{PhD}^{4}$

${ }^{1}$ Nottingham University Hospitals NHS, Fetal Medicine Unit, Nottingham, United Kingdom

${ }^{2}$ Icahn School of Medicine at Mount Sinai, Department of Obstetrics, Gynecology, and Reproductive Science, New York, New York

${ }^{3}$ NYU School of Medicine, Department of Obstetrics and Gynecology, New York, NY, USA

${ }^{4}$ Fetal Medicine Unit, St George's University Hospitals NHS Foundation Trust, London, UK and Molecular \& Clinical Sciences Research Institute, St George's University of London, UK

\section{Corresponding author}

Dr Andrea Kaelin Agten

Nottingham University Hospitals NHS

Queens Medical Centre

Fetal Medicine Unit

Derby Road

Nottingham, NG7 2UH

UK

a.kaelin@gmail.com

Short title: CSP-Registry

Keywords: accreta, cesarean section, uterine scar 
Cesarean scar pregnancy (CSP) is a potentially dangerous, iatrogenic consequence of a previous cesarean section (CS). If missed or inadequately managed, CSP can lead to severe maternal complications in any trimester of pregnancy. ${ }^{1}$

\section{Pathophysiology of CSP}

A CSP occurs when the pregnancy implants on the uterine scar, usually from a previous CS. It is suggested that decidual defect following the iatrogenic creation of a CS scar has an adverse effect on early implantation. There are several risk factors discussed in the literature that may predispose persistent uterine scar defects after CS. The impact of single- versus double-layer closure of the uterine incision on scar defects is still unknown. Sardo et al. concluded in his systematic review that the incidence of Scar defects and uterine dehiscence in a subsequent pregnancy are similar for single versus double layer closure following a $\mathrm{CS}^{2}$. In contrast, Bij de Vate et al. suggested that single-layer closure might be a risk factor for developing a niche ${ }^{3}$. Of interest is also the impact of cervical dilatation on the healing of the scar. In a study with 409 women, the residual myometrial thickness over the scar was significantly reduced in women that had a cesarean section performed in the second stage of labour as compared to first stage of labour ${ }^{4}$. A thin myometrium is significantly associated with the occurrence of scar defects ${ }^{4}$. In another large cohort study of 371 women, the following were risk factors for scar defects: gestational diabetes with an OR 1.73 (95\% Cl 1.02-2.92, $\mathrm{P}=.042$ ), previous CS OR 3.14 ( $95 \%$ C.I. 1.90-5.17; $\mathrm{P}<.001$ ), and advanced maternal body mass index 1.06 (95\% C.I. 1.01-1.11; P = .012) ${ }^{5}$. In a large 
Chinese study with 514 women, perioperative fever and infection were also associated with scar defects ${ }^{6}$.

Presumably, the implantation of the conceptus in a subsequent pregnancy into the uterine niche/scar is a random occurrence and leads to the development of CSP.

\section{Prevalence of CSP}

There is a wide range in the prevalence of scar niches described in the literature. In a random population of women with previous CS, the prevalence of CSP was found to vary between $56 \%$ and $84 \%$, using contrast-enhanced sonohysterography ${ }^{3}$. The estimated prevalence of CSP is 1 in 1500/2000 women with previous CS ${ }^{1}$. Looking into placenta accreta, there is a wide variation in reported prevalence, ranging between 1 in 300 and 1 in 2000 pregnancies $^{7}$. In a systematic review including 14 cohort studies with 3889 pregnancies found a pooled incidence of placenta previa accreta of $4.1 \%$ in women with 1 prior cesarean and $13.3 \%$ in women with $\geq 2$ previous cesarean deliveries. Even though the overall prevalence of CSP is very low, the incidence is significantly increased when both CS in the previous pregnancy and a low-lying placental position in the current pregnancy are diagnosed.

\section{Natural history of CSP}

A number of early, 1st trimester CSPs will spontaneously demise much like some intrauterine and ectopic gestations. Some patients may select ending the pregnancy after being counseled about the possibility of serious complications in the second and 
third trimester. If the pregnancy is continued and abnormal invasion of the placenta (AIP) develops (Figure 1), these pregnancies may lead to significant and serious maternal morbidity such as vaginal or intraabdominal bleeding, uterine rupture and shock. In a systematic review of 17 studies of 69 cases of CSP managed expectantly, $13 \%$ experienced an uncomplicated miscarriage, uterine rupture during the first or second trimester of pregnancy occurred in $10 \%$ and $77 \%$ of women progressed to the third trimester of pregnancy - of whom 39\% experienced severe bleeding with a high rate of AIP diagnosis ${ }^{8}$. Despite the maternal morbidity associated with continuing a CSP, the majority ( $75 \%)$ of pregnancies complicated by CSP may progress to term and result in live birth, thus questioning whether early termination of pregnancy should be the only therapeutic option offered to these women.

\section{Diagnosis of CSP and AIP}

The best imaging modality to diagnose a CSP is transvaginal ultrasound (US) ${ }^{9}$. The diagnostic criteria include an empty uterine cavity, an empty and closed endocervical canal and the visualization of a gestational sac implanted anteriorly in the area of the scar, at the level of the internal os, negative sliding organ sign (gentle pressure with the vaginal probe does not displace the gestational sac from its position), and increased placental vascularity (typically with high blood flow velocity $20 \mathrm{~cm} / \mathrm{s}$ and low impedance $\mathrm{Pl}<1)^{1,9}$. It is very important to record the position of the placenta and gestational sac. Jurkovic showed before that vascularity of the pregnancy on Doppler examination and gestational age were the most significant predictors of excessive 
blood loss ${ }^{10}$. An early diagnosis of a CSP allows timely management. Missed diagnoses of CSP reported in the literature are high. In a review, of 751 cases of CSP $107(13.6 \%)$ were missed or misdiagnosed resulting in a large number of bleeding complications, some as severe requiring hysterectomy. Signs of abnormal invasion of the placenta can already be detected in the first trimester of pregnancy. Kaelin Agten showed that CSP implanted in a defect scar resulted in more severe AIP and Cali showed that the relationship between the gestational sac and the endometrial line (cross-over sign) in CSP was useful in predicting the evolution of CSP towards different types of AIP 11,12 . Ultrasound has a good diagnostic accuracy to detect an abnormally invasive placenta (AIP) in the first trimester. At least one US sign suggestive of AIP was detected in $83.7 \%(95 \% \mathrm{Cl} 61.3-97.5)$ of cases with the confirmed diagnosis ${ }^{13}$. US signs of AIP are also used to describe CSP, as the are from the same histopathologically disease spectrum ${ }^{14}$. According to Jauniaux, the incidence of AIP was $4.1 \%$ in women with 1 prior cesarean and $13.3 \%$ in women with $\geq 2$ previous CS $^{15}$. Therefore, screening for AIP in early pregnancy is recommended ${ }^{16}$.

\section{Management of CSP}

To date, there is no standardized treatment protocol agreed or available ${ }^{17}$. Surgical approaches for pregnancy interruption include dilatation \& curettage, laparoscopy, hysteroscopy or laparotomy. Medical therapies may be affected with systemic administration of methotrexate or ultrasound-guided local intragestational sac injection of methotrexate or potassium chloride. However, the success rate of the latter in 
avoiding serious maternal complications with a fetal heartbeat is uncertain. As a result, occasionally physicians advocate an interventional radiology approach to embolize a CSP. In many centers, a conservative approach is adopted often inadvertently through missed diagnosis or intentionally through maternal choice for a much-wanted pregnancy despite the risks of maternal morbidity.

\section{Justification for an international registry}

There is general consensus that a low implantation in a uterus previously scarred by CS predisposes to CSP. Physicians also acknowledge that even though a rare diagnosis, CSP results in early pregnancy uterine rupture/hemorrhage or term AIP with significant maternal morbidity. The lack of agreed clinical screening protocols and relatively infrequent US diagnosis result in a paucity of data regarding the true natural history and data to support the optimal management of this condition. What data is available and published come from small retrospective clinical series or those that are influenced by referral bias. Consequently, there is wide variation in practice in the management of CSP from routine early termination of pregnancy to expectant management and planned Cesarean hysterectomy in the late third trimester.

We propose and funded an international CSP registry to prospectively collate the diagnosis, natural history, variation in surgical/medical practice, maternal complications and pregnancy outcome. Cases can be uploaded by participating doctors upon registration on www.csp-registry.com (Figure 2). We invite doctors with 
CSP cases to upload pregnancy data onto the registry in order to facilitate collation of knowledge and produce the best evidence for diagnosis and treatment of CSP.

The authors declare no conflict of interest 


\section{References}

1. Timor-Tritsch IE, Monteagudo A. Unforeseen consequences of the increasing rate of cesarean deliveries: early placenta accreta and cesarean scar pregnancy. A review. Am J Obstet Gynecol 2012; 207: 14-29.

2. Di Spiezio Sardo A, Saccone G, McCurdy R, Bujold E, Bifulco G, Berghella V. Risk of Cesarean scar defect following single- vs double-layer uterine closure: systematic review and meta-analysis of randomized controlled trials. Ultrasound Obstet Gynecol 2017; 50: 578-583.

3. Bij de Vaate AJ, van der Voet LF, Naji O, Witmer M, Veersema S, Brolmann HA, Bourne T, Huirne JA. Prevalence, potential risk factors for development and symptoms related to the presence of uterine niches following Cesarean section: systematic review. Ultrasound Obstet Gynecol 2014; 43: 372-382.

4. Pomorski M, Fuchs T, Rosner-Tenerowicz A, Zimmer M. Standardized ultrasonographic approach for the assessment of risk factors of incomplete healing of the cesarean section scar in the uterus. Eur J Obstet Gynecol Reprod Biol 2016; 205: 141-145.

5. Antila-Langsjo RM, Maenpaa JU, Huhtala HS, Tomas El, Staff SM. Cesarean scar defect: a prospective study on risk factors. Am J Obstet Gynecol 2018; 219: 458.e451-458.e458.

6. Pan H, Zeng M, Xu T, Li D, Mol BWJ, Sun J, Zhang J. The prevalence and risk predictors of cesarean scar defect at 6 weeks postpartum in Shanghai, China: A prospective cohort study. Acta Obstet Gynecol Scand 2019; 98: 413-422.

7. Jauniaux E, Alfirevic Z, Bhide AG, Belfort MA, Burton GJ, Collins SL, Dornan S, Jurkovic D, Kayem G, Kingdom J, Silver R, Sentilhes L. Placenta Praevia and Placenta Accreta: Diagnosis and Management: Green-top Guideline No. 27a. BJOG 2018. DOI:10.1111/1471-0528.15306.

8. Cali G, Timor-Tritsch IE, Palacios-Jaraquemada J, Monteaugudo A, Buca D, Forlani F, Familiari A, Scambia G, Acharya G, D'Antonio F. Outcome of Cesarean scar pregnancy managed expectantly: systematic review and meta-analysis. Ultrasound Obstet Gynecol 2018; 51: 169-175.

9. Harb HM, Knight M, Bottomley C, Overton C, Tobias A, Gallos ID, Shehmar M, Farquharson R, Horne A, Latthe P, Edi-Osagie E, MacLean M, Marston E, Zamora J, Dawood F, Small R, Ross J, Bourne $T$, Coomarasamy A, Jurkovic D. Caesarean scar pregnancy in the UK: a national cohort study. BJOG 2018; 125: 1663-1670.

10. Jurkovic D, Knez J, Appiah A, Farahani L, Mavrelos D, Ross JA. Surgical treatment of Cesarean scar ectopic pregnancy: efficacy and safety of ultrasound-guided suction curettage. Ultrasound Obstet Gynecol 2016; 47: 511-517.

11. Cali G, Forlani F, Minneci G, Foti F, Di Liberto S, Familiari A, Scambia G, D'Antonio F. Firsttrimester prediction of surgical outcome in abnormally invasive placenta using the cross-over sign. Ultrasound Obstet Gynecol 2018; 51: 184-188.

12. Kaelin Agten A, Cali G, Monteagudo A, Oviedo J, Ramos J, Timor-Tritsch I. The clinical outcome of cesarean scar pregnancies implanted "on the scar" versus "in the niche". Am J Obstet Gynecol 2017; 216: 510.e511-510.e516.

13. Cali G, Forlani F, Foti F, Minneci G, Manzoli L, Flacco ME, Buca D, Liberati M, Scambia G, D'Antonio F. Diagnostic accuracy of first-trimester ultrasound in detecting abnormally invasive placenta in high-risk women with placenta previa. Ultrasound Obstet Gynecol 2018; 52: 258-264. 14. Timor-Tritsch IE, Monteagudo A, Cali G, Vintzileos A, Viscarello R, Al-Khan A, Zamudio S, Mayberry $\mathrm{P}$, Cordoba MM, Dar P. Cesarean scar pregnancy is a precursor of morbidly adherent placenta. Ultrasound Obstet Gynecol 2014; 44: 346-353. 
15. Jauniaux E, Bhide A. Prenatal ultrasound diagnosis and outcome of placenta previa accreta after cesarean delivery: a systematic review and meta-analysis. Am J Obstet Gynecol 2017; 217: 2736.

16. Panaiotova J, Tokunaka M, Krajewska K, Zosmer N, Nicolaides KH. Screening for morbidly adherent placenta in early pregnancy. Ultrasound Obstet Gynecol 2019; 53: 101-106.

17. D'Antonio F, Palacios-Jaraquemada J, Lim PS, Forlani F, Lanzone A, Timor-Tritsch I, Cali G. Counseling in fetal medicine: evidence-based answers to clinical questions on morbidly adherent placenta. Ultrasound Obstet Gynecol 2016; 47: 290-301. 


\section{Figure legends}

Figure 1. Evolution of a cesarean scar pregnancy.

Figure 2. QR code to access CSP-registry 


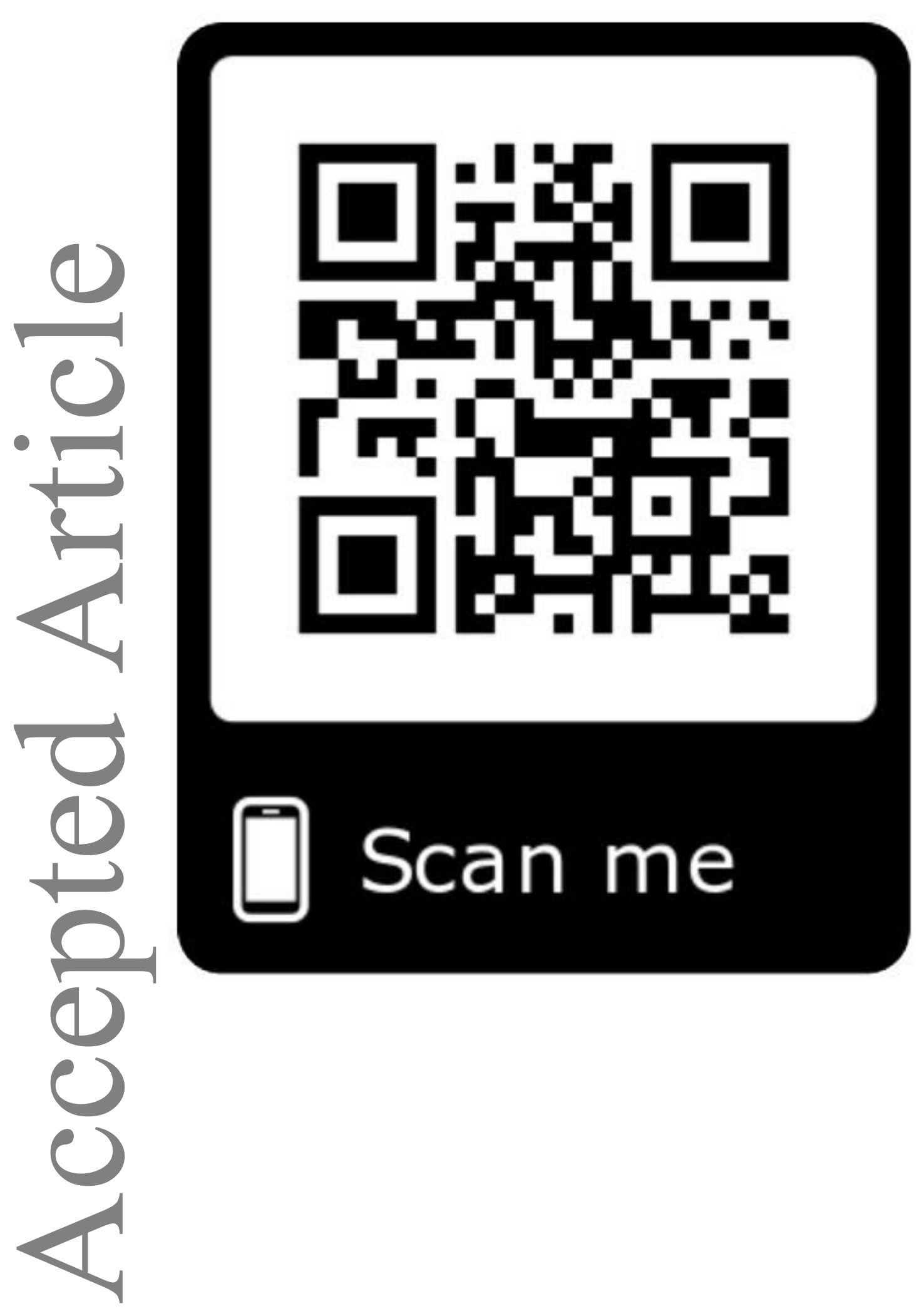

